\title{
Real-time Lighting Design for Interactive Narrative
}

\author{
Magy Seif El-Nasr, Ian Horswill \\ Northwestern University \\ Computer Science Department \\ 1890 Maple Avenue \\ magy@northwestern.edu, ian@cs.northwestern.edu
}

\begin{abstract}
Lighting design is an important element of scene composition. Designers use lighting to influence viewers' perception by evoking moods, directing their gaze to important areas, and conveying dramatic tension. Lighting is a very time consuming task; designers typically spend hours manipulating lights' colors, positions, and angles to create a lighting design that accommodates dramatic action and tension. Such manual design is inappropriate for interactive narrative, because the spatial and dramatic characteristics of an interactive scene, including dramatic tension, camera location, and character actions, change unpredictably, necessitating continual redesign as the scene progresses. In this paper, we present a lighting design system, called ELE (Expressive Lighting Engine), that automatically, in real-time, adjusts angles, positions, and colors of lights to accommodate the dramatic and spatial characteristics of a scene, while conforming to the established style and ensuring visual continuity. ELE uses constraintbased non-linear optimization algorithms to configure lights using cost functions formulated based on traditional film and theatrical lighting design theory.
\end{abstract}

\section{Introduction}

Lighting designers rely on lighting to visually compose a scene. Lighting is essential not only for establishing visibility, but also for emphasizing visual tension, establishing depth, directing viewers' gaze to important objects/characters/areas, and providing mood [1-3]. Film Noir, for example, is distinguished for employing high contrast lighting to project mystery and visual tension. These qualities are significant for interactive narrative, because they influence the participant's action, engagement, and mood [4]. 
Creating a lighting design that satisfies these goals for an interactive scene is a difficult problem. In a typical interactive narrative, player's choices and movements affect the camera angle and position as well as the characters' orientations, positions, and relationship values, and thus, influence dramatic intensity and action. Interactive entertainment productions currently use statically designed lighting, which is context insensitive and often result in partially lit or unlit characters (e.g. The Thing) leading to player's frustration.

Lighting design is a complex problem. Lighting designers have identified several lighting design goals and constraints, and developed many rules to satisfy them [2, 5-8]. In many cases, however, these goals conflict, therefore, lighting design as any design process involves many tradeoffs. For instance, establishing visibility for all objects in a scene often conflict with providing a clear visual focus or achieving intense and dark moods, because creating such moods require high contrast and less visibility [9]. Additionally, continually adjusting light angles and positions for visibility may cause distracting changes, and thus conflict with visual continuity.

This paper presents a lighting system, called ELE (Expressive Lighting Engine), which introduces a new approach to lighting design for interactive entertainment. As an alternative to static lighting, ELE automatically adjusts lighting angles, positions, and colors, to satisfy the lighting design goals and accommodate camera's and characters' positions and orientations, while maintaining visual continuity. Recognizing lighting design as an optimization problem that involves balancing many goals, ELE uses nonlinear constraint optimization algorithms. In addition, the system adapts theories discussed by Calahan [1] and the traditional lighting design theory addressed by filmmakers and theatre designers [2, 5, 7]. ELE has been implemented and tested within several scenes from Mirage - an interactive story that we developed based on the Greek Tragedy Electra.

In this paper, we will first review some of the current techniques used in interactive entertainment productions, and outline some of the related research in the field. We will then discuss ELE in detail outlining its components. We will then conclude by discussing summary of contribution and future research.

\section{Related Research}

Interactive entertainment productions use static manually designed lighting, e.g. set-based or ambient ${ }^{1}$ lighting. Ambient lighting, used in many games, including the Sims and Sim city, is simple and does not require adjustments as the characters move or the shot changes. However, it has no cinematic quality and serves no dramatic or aesthetic

\footnotetext{
${ }^{1}$ The term ambient lighting is used here to define a light design where every object in the scene is lit equally using a constant intensity, and where the light has no direction. [10] T. Moller, and Haines, E., Real-time Rendering. MA: A K Peters, Ltd, 1999.
} 
functions [11]. To establish mood and visual focus, first person and adventure game ( e.g. Max Payne and Devil May Cry) designers use set-based lighting, where they manually set positions, orientations, and colors for each light in the scene depending on the set design $[4,12]$. While this scheme provides a workable solution for most games, it is inflexible due to the following reasons. First, it cannot be used to provide character modeling - a technique where more lights are added around a character to show depth and texture. Second, in some situations the character may not be sufficiently lit; a problem that surfaces in games such as the Thing. Third, it does not adequately accommodate the dynamic dramatic tension and visual focus.

Few research projects proposed an alternative approach to scripting or manually designed visuals (such as lighting or camera movement). M. He et al. used film-based cinematography techniques to guide an authored interactive narrative [13]. They, however, did not address lighting. Tomlinson proposed a system that manipulates light colors and camera movements to present the user with an interpretation of the world based on the characters' internal states [14]. For example, it uses a low camera angle to show that a character is powerful or harsh red light to make a character look demonic. In his work, the function of the camera and lighting was restricted to portraying the emotional states of characters in a scene, and thus visual design goals and their link to the narrative as addressed by filmmakers and theatre designers were not addressed [15].

\section{ELE (Expressive Lighting Engine)}

ELE is designed to automatically set the number of lights used, their positions, colors, and angles based on rules formulated using theatre and film lighting design theory. ELE sits on top of a real-time rendering engine and is used to provide a level of abstraction from the low-level details of manipulating and placing lights individually through graphic engines. Thus, artists no longer have to script each light change in the scene, and ensure that these changes follow a general continuum between frames.

I assume that there exists a system that passes several parameters to ELE, including a set of parameters describing style, local light sources, stage configuration and dimensions, characters' dimensions, dramatic focus (the area/characters towards which attention should be directed) and the dramatic intensity of the situation. Using these parameters ELE computes the number of lights used. For each of these lights, it computes the type of instrument (e.g. spot light or point light), color in RGB color space, attenuation, position as a $3 \mathrm{D}$ point, orientation including the facing and up vectors, range, masking parameters, and, depending on the light instrument used, the Penumbra and Umbra angles. These parameters are given to a rendering engine to render the frame.

ELE is composed of three subsystems: a system that determines the number of lights used and the areas they affect, a system that selects a color for each light in the scene, and a system that selects an azimuth and elevation angles for each light in the scene. In 
addition, ELE interacts with the camera system to synchronize lighting changes with camera movement and to ensure that distracting changes are hidden between camera cuts, since Neuroscience and psychology literature have found that human vision does not perceive changes made between camera cuts [18]. I will describe these systems next.

\subsection{Allocating Lights}

Lights are expensive. Rendering time is proportional to the number of lights used. Most often rendering engines limit the number of lights used to achieve real-time rendering speed (30 frames/second). For instance, Wildtangent (a publicly available rendering engine) restricts the number of lights to eight lights. However, artists use many lights to model characters (i.e. show depth and texture of characters) and establish depth. Lighting designers at Pixar, for example, often use as many as thirty two lights to light one scene. ELE finds a compromise using optimization.

Following film and theatre lighting design theory, ELE finds a configuration of cylindrical areas, $a_{i}$, such that the visible area, $V$, is covered satisfying several constraints, including the maximum number of lights (which is limited considering the speed needed), desired visibility of objects and their whereabouts, the desired visual focus (dramatic focus), desired modeling for each character, and desired depth, all of which are determined depending on the given style and situation.

To establish depth, ELE differentiates between areas in the foreground, middle ground, and background. It creates character areas, such that all characters are assigned an area, as follows:

- For each character $c$ create a new area $a$ and assign $c$ to it

- For each area $a$, if $\exists a^{\prime}$ s.t. $\left|a-a^{\prime}\right|<\varepsilon$, and both are focus (or non-focus) areas then merge $a, a^{\prime}$

It then finds an allocation $p$, where $p: L \rightarrow A$ is an assignment of lights to areas, maximizing an objective function composed of the lighting design goals described above:

$$
p_{\text {opt }}=\arg \max _{p}\left(\lambda_{v} V(p)+\lambda_{d} D(p)+\lambda_{m} M(p)+\lambda_{c} C(p)\right),
$$

where $\mathrm{V}(p)$ is the percentage of visible areas that are assigned lights, $\mathrm{M}(p)$ is the average number of lights assigned to character areas (to ensure desired modeling), $\mathrm{D}(p)$ is depth measured by the difference between the number of lights assigned to the background and foreground areas, and $\mathrm{C}(p)$ is visual continuity measured by the difference of the configuration $p$ from the one used in the previous frame. The weights are set by default rules developed based on traditional lighting design theory. 


\subsection{Configuring Angles}

ELE selects azimuth and elevation angles for each light in the scene, including key, fill, and backlights. According to Film and theatre lighting design, designers select angles for each light in the scene to conform to several requirements: ensuring visual continuity, maintaining the illusion of a light source established direction, provoking mood, establishing the desired modeling, and providing visibility.

These goals/requirements conflict; for example, an angle that provides best visibility may hinder modeling or mood. ELE balances these goals using a nonlinear optimization algorithm based on hill climbing. It selects a key light azimuth angle by optimizing a multi-objective cost function, where the objective functions correspond to the lighting design goals, as follows:

$$
\begin{aligned}
& \operatorname{cost}\left(k, k^{-}, m\right)= \\
& \lambda_{v}(1-V(k))+\lambda_{-}\left|k-k^{-}\right|+\lambda_{m_{a}}\left|k-m_{a}\right|+\lambda_{l} \min _{i}\left|k-l_{i}\right|
\end{aligned}
$$

Where $k$ is the key light angle, $k^{-}$is the key light angle from the previous frame, and $m_{a}$ is the mood angle azimuth suggested by the artist or calculated by ELE (using a set of default rules based on mood angles addressed by [9]), $\lambda_{v}$ is the cost of deviation from an orientation that establishes best visibility and modeling, $\lambda$. is the cost of changing the key light angle over time (to enforce visual continuity), $\lambda_{l}$ is the cost of deviation from an established light source (e.g. torch, window) angle/direction. In addition, for each light source, and $l_{i}$ is the angle of light from the light source $i$ on the subject or area in question, and $\lambda_{m_{a}}$ is the cost of deviation from an angle that shows a specific mood.

Thus, the cost function in (1.2) calculates the best angle given the value of the angle $\mathrm{k}$ relative to the objectives desired and their importance projected by the weights. The term $\left|k-k^{-}\right|$calculates visual continuity by calculating the difference between the angle selected and the one selected in the previous frame. The third term, $\left|k-m_{a}\right|$ calculates the difference between the angle selected and the angle suggested by ELE or the artist for achieving the mood desired. Thus, the third term ensures that the right mood is provided in the given shot. The last term, $\min \left|k-l_{i}\right|$, calculates the difference between the angle of light selected and the angle of light emitted by the nearest practical source. $V(k)$ measures the quality of the key light azimuth angle relative to visibility and modeling following Millerson's guidelines [5], as follows:

$$
V(k, s)=\sin (k) \cos (s),
$$

where $k$ is the angle between the key light and the camera, and $s$ is the angle between the nose light and the key light. 
Similarly, ELE uses the following multi-objective cost function to find the best key light elevation angle:

$$
\cos t\left(e, m_{e}\right)=\lambda_{m_{e}}\left|e-m_{e}\right|+\lambda_{l} \min _{i}\left|e-l_{i}\right|
$$

where $e$ is the elevation angle, $m_{e}$ is the mood elevation angle suggested by the artists or computed by ELE (using rules documented by [9]), $l_{i}$ is the elevation of the practical source $i, \lambda_{m_{e}}$ is the cost associated mood elevation angle, and $\lambda_{l}$ is the cost of ensuring that the elevation angle conforms to the elevation of the practical source angle. ELE uses gradient descent to find the best $e$ that minimizes the cost function above.

Fill and backlight azimuth angles are calculated depending on the value of the key light angle and the angle between the camera and the subject. According to the guidelines described by Millerson [5], fill light azimuth and elevation angles are calculated to be the mirror image of the key light angle. Backlight azimuth angle is calculated as follows:

$$
b=(k-c+\pi) \bmod 2 \pi
$$

where $k$ and $c$, again, are the are the respective angles of the key light and camera. Backlight elevation angle is set to $45^{\circ}$ as recommended by [5].

\subsection{Color Optimization System}

Colors of lights in a scene compose the contrast and feeling of the entire image. A change of one light's color may affect the entire image. Therefore, ELE manipulates the colors of light evaluating each color change and its effect on the whole image. ELE evaluates color using several parameters, including desired contrast, importance of visual continuity, color palette constraints, as well as ideal values for the hue, saturation, lightness, warmth/coolness for each light, and costs describing the importance of adhering to these values. Using default rules formulated based film and theatre lighting theory, ELE determines default values for these parameters given the style, dramatic intensity, dramatic focus, and dramatic situation.

Using the ideal values and their associated costs, ELE uses nonlinear optimization that searches through a nine-dimension space of RGB values differentiating between colors of focus, non-focus, and background areas to select a color for each individual light in the scene evaluating this color using a multi-objective cost function defined as follows:

$$
\begin{aligned}
& \operatorname{cost}\left(c^{t}, c^{t-1}\right)=\lambda_{d}\left(D\left(c^{t}\right)-d\right)^{2}+\lambda_{c}\left(\operatorname{contrast}_{\phi}\left(c^{t}\right)-\delta\right)^{2}+v(x)+ \\
& \sum_{i \in\{f, n, b\}} P\left(c_{i}^{t}, c_{i}^{t-1}\right), \\
& \text { where } p\left(c_{i}^{t}, c_{i}^{t-1}\right)=\begin{array}{l}
\lambda_{s_{i}}\left(S\left(c_{i}^{t}\right)-s_{i}\right)^{2}+\lambda_{h_{i}}\left(H\left(c_{i}^{t}\right)-h_{i}\right)^{2}+ \\
\lambda_{l_{i}}\left(L\left(c_{i}^{t}\right)-l_{i}\right)^{2}+\lambda_{w_{i}}\left(W\left(c_{i}^{t}\right)-w_{i}\right)^{2}+\lambda_{c h} E\left(c_{i}^{t}, c_{i}^{t-1}\right),
\end{array}
\end{aligned}
$$


and where $c^{t}$ is a vector of light colors for focus, $f$, non-focus, $n$, and background, $b$, areas at frame $t$. Color $c_{i}^{t}$ is represented in RGB color space, S(c) denotes the saturation of color $c, \mathrm{H}(c)$ denotes the hue of color $c, \mathrm{~L}(c)$ denotes lightness of color $c$ (in RGB color space). ELE uses CIEDE2000 - a well known formula for measuring color difference [16, 17]. The depth, $D(c)$, of a color vector, $c$, is defined as the color difference between colors of lights lighting the background areas and those lighting other areas.

Following traditional lighting design theory, contrast is used to establish visual tension that parallels the dramatic tension in the scene [2]. Using the guidelines documented by filmmakers and designers, contrast is defined relative to the dramatic focus of the scene [2] as the difference between colors of lights lighting the focus area and a weighted sum of the colors lighting the other areas as follows:

$$
\text { contrast }_{\phi}(c)=\sum_{i \neq \text { focus }} w_{i}\left|\phi\left(c_{\text {focus }}\right)-\phi\left(c_{i}\right)\right|
$$

where $\phi$ is the color component (lightness, warmth, or saturation) over which we're computing contrast, $c$ is a vector of the light colors, $\mathrm{c}_{i}$ is a color for an area type $i$, where $i \in\{$ focus, non - focus, background $\}$ and focus is the index of the dramatic focus area.

The optimization problem discussed above is a constraint-based optimization problem, where the color, $c$, is constrained to specific values. ELE uses a boundary method to bind the feasible solutions using a barrier function that approaches $\infty$ as the values approach the boundary defined by the feasibility region. Although gradient descent has major drawbacks, including occurrence of oscillations and being easily stuck in a local minimum, ELE uses gradient descent because a local minimum in our case is preferable since it provides a solution closer to the previous one.

\section{Results}

ELE was implemented and tested within several scenes from an interactive story, called Mirage - an interactive drama based on the Greek Tragedy Electra. ELE was used to make real-time lighting design decisions that adapt to the story and the situations presented. ELE adapts the lighting to the scene's dramatic action and tension maintaining the established style and visual continuity.

Figures 1 and 2 show some screenshots comparing ELE to other techniques. Figure 1 compares two screenshots from Mirage where Electra unsheathes a sword ready to attack the user, and where one screenshot was rendered using a static lighting approach and the other using ELE. As shown in the figure, ELE provides a better depiction of the dramatic moment. To evaluate the performance of ELE in accommodating movements, figure 2 compares set-based lighting and dynamic lighting used by ELE in a shot where the character and the camera made unanticipated movements. As shown, set-based lighting results in partially lit characters due to character movement and changes in the camera 
view. On the other hand, ELE ensures visibility by dynamically adapting to the movements, as shown.

Figure 3 presents a sequence of screenshots showing ELE adapting the lighting in a scene to accommodate movement and rise in tension. For example, in the moment after the choice point, ELE accommodated the steep rise in dramatic tension by first requesting a cut from the camera system, and then changing the color contrast within the cut, thus maintaining visual continuity. Comparing the contrast before the choice point to the contrast after the choice point, one can see the dramatic difference. However, viewers were not distracted by this steep change, since it was done within a camera cut.

To evaluate the performance of ELE as a lighting designer, a web-based evaluation was conducted. We put the website up for 7 days and asked many visual artists to visit the site and conduct the evaluation test. The reviewers were first asked to view three movies of the same scene lit by (1) ELE, (2) ambient lighting, and (3) statically designed lights. The viewers were allowed to switch between movies, stop, pause, rewind, or forward any of the movies at any time. When ready to begin answering the questionnaire, reviewers can just press on the questionnaire button. (They can go back to the movies at any time). Reviewers where then asked ten questions comparing the scenes in terms of the lighting design goals (such as providing depth, visual focus, etc). Since the test used the same models and scene content, the variables evaluated were constrained to only lighting. ELE rated higher than the other approaches.

\section{Conclusion \& Future Work}

Lighting is an important element of scene composition. Film and theatre lighting designers have long realized the importance of lighting in enhancing and enriching the experience. In this paper, we presented ELE - an Expressive Lighting Engine that follows traditional film, theatre, and animation techniques to provide a new lighting design

technique that is more suited for interactive scenes, where dramatic and spatial content unpredictably changes. Due to the complexity of the lighting process and limitations of real-time rendering engines, we left several topics for future research, including shadows, interrelflections, and refractions.

\section{References}

[1] S. Calahan, "Storytelling through lighting: a computer graphics perspective," presented at Siggraph Course Notes, '96.

[2] B. Block, The Visual Story: Seeing the Structure of Film, TV, and New Media. New York: Focal Press, '01.

[3] P. Ward, Picture Composition for Film and Television. Oxford: Focal Press, '96. 
[4] D. Carson, "Environmental Storytelling: Creating Immersive 3D Worlds Using Lessons Learned from the Theme Park Industry," in Gamasutra, '00.

[5] G. Millerson, The Technique of Lighting for Telivision and Film, 3rd ed. Oxford: Focus Press, ' 91.

[6] D. Viera, Lighting for Film and Electronic Cinematography. Belmont: Wadsworth Publishing Company, '93.

[7] R. Lowell, Matters of Light and Depth. New York: Lowel-light Manufacturing, Inc., '92.

[8] B. Foss, Filmmaking: Narrative \& Structural Techniques. Los Angeles: Silman-James Press, '92.

[9] D. Campbell, Technical Theatre for Non-technical People: Allworth Press, '99.

[10] T. Moller, and Haines, E., Real-time Rendering. MA: A K Peters, Ltd, '99.

[11] J. Birn, "Digital Lighting \& Rendering," G. Maestri, Ed. Indianapolis: New Riders, '00.

[12] A. Maattaa, "GDC 2002:Realistic Level Design for Max Payne," in Gamasutra, '02.

[13] M. C. L. He, and D. Salesin, "The Virtual Cinematographer: A Paradigm for Automatic Real-time Camera Control and Directing," presented at International Conference on Computer Graphics and Interactive Techniques, '96.

[14] W. M. Tomlinson, "Interactivity and Emotion through Cinematography." MIT Media Lab: MIT, '99.

[15] D. C. a. A. Knoph, The Book of Movie Photography. London: Alfred Knopf, '79.

[16] B. H. Hill, Roger, T. , and Vorhagen, F. W., "Comparative Analysis of the Quantization of Color Spaces on the Basis of the CIELAB Color-Difference Formula," ACM Transactions on Graphics, vol. 16, pp. 109-154, '97.

[17] M. R. Luo, Cul, G., and Rigg, B., "The Development of CIE 2000 Colour Difference Formula: CIEDE2000," vol. 2000: CIELAB, '00.

[18] H. a. Hollingworth, "Global transsaccadic change blindness during scene perception," Psychological Science, in press.
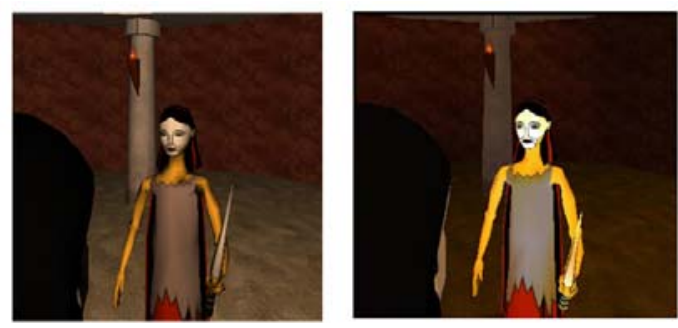

Fig. 1. A screenshot from Mirage where Electra unsheathes the sword to attack Archemedis. The two images compare renderings of the same scene using ELE (right), camera fill (left) 

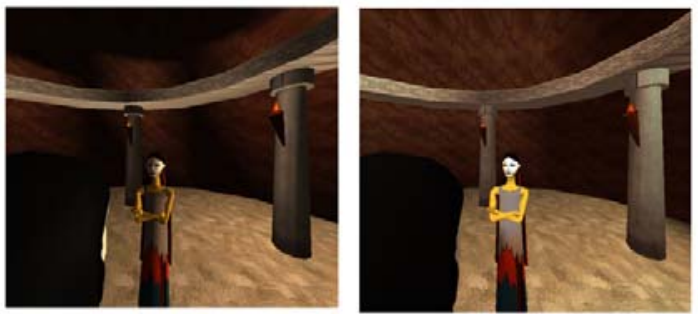

Fig. 2. Shows two screenshots using ELE (right), camera fill (left)
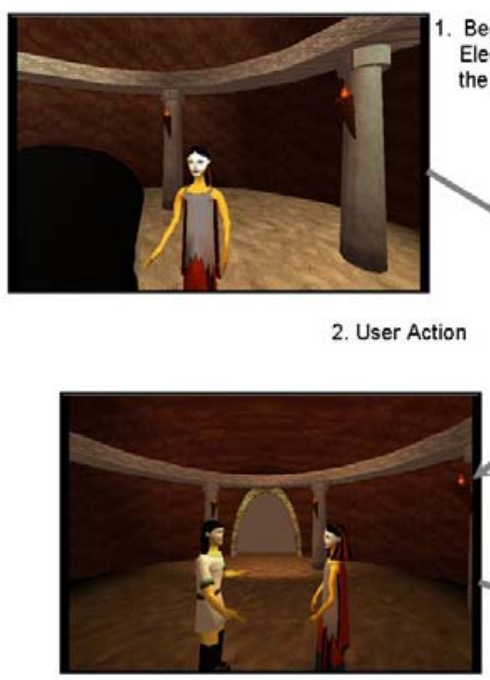

Beginning of the Scene,

Electra tells user about

the plague that is talking over the kingdom

4. Later on, tension increases (lighting: contrast increases)

After making the choice, tension rises (lighting: asked for camera cut, within the cut ELE increased contrast to match the tension)

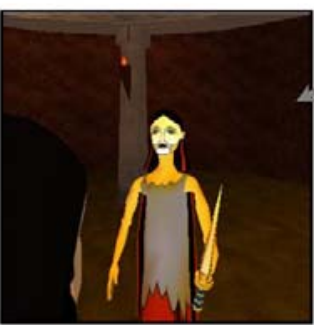

At the peak of the dramatic tension;

(lighting: Contrast increases

ELE under lights Electra to show

her as sinister \& emphasize action -

a rule used from Campbell (99))

Fig. 3. Screenshots from Scene 8C 